MS031.004

Microsymposium

\title{
Crystallographic investigation of metallic and bimetallic nanoparticles
}

Oleq Prymak ${ }^{1}$, Kateryna Loza ${ }^{1}$, Viktoria Grasmik ${ }^{1}$, Kevin Pappert ${ }^{1}$, Alexander Rostek ${ }^{1}$, Jens Helmlinger ${ }^{1}$, Martin Gocyla ${ }^{2}$, Marc Heggen ${ }^{2}$, Matthias Epple ${ }^{1}$

${ }^{1}$ Inorganic Chemistry, University Of Duisburg-Essen, Essen, Germany, ${ }^{2}$ Ernst Ruska-Centre and Peter Grünberg Institute / Forschungszentrum Jülich GmbH, Jülich, Germany

E-mail: oleg.prymak@uni-due.de

Metallic nanoparticles represent a well-established part of nanoscience today due to their tunable physico-chemical properties and their potential in nanomedicine. Especially noble metals and their combination in nanoalloys, including core-shell structures, have attracted high interest, with silver, gold, platinum, and palladium nanoparticles as most prominent examples. Due to the antibacterial effects $(\mathrm{Ag})$, the easy covalent functionalization by thiol or phosphane chemistry (Au) and inherent catalytic properties (Pt, Pd), their applications are manifold. We have synthesized PVP-coated monometallic (Ag, Au, $\mathrm{Pt}, \mathrm{Pd}$ ) and bimetallic $\mathrm{AgAu}$ and $\mathrm{AgPt}$ nanoparticles by wet chemical co-reduction, including bimetallic core-shell nanostructures. Spherical and shaped ( $\mathrm{Ag}$, platelets, cubes, and rods) nanoparticles in a size range of 5-50 nm were produced and characterized by colloid-chemical (DCS), microscopic (SEM, HRTEM) and spectroscopic (EDX, UV-vis) methods. By extended crystallographic investigation using X-ray powder diffraction (XRD) with different geometrical setups including grazing incidence diffraction and pole figure analysis with subsequent Rietveld refinement, a precise determination of the lattice parameters, the crystallite size and the microstrain as well their preferred orientation as a function of morphology was performed. X-ray powder diffraction is well suited to describe not only the crystallographic identity of nanopatricles, but also their size and anisotropic morphology with further information about their domain nature: single or polycrystalline.

[1] Helmlinger, J. et al. (2016). Cryst. Growth Des. 16, 3677-3687.

[2] Ristig, S. et al. (2015). J. Mater. Chem. B3, 4654-4662.

[3] Banerjee, S. et al. (2014). Chem. Mater. 26, 951-957.

Keywords: noble metals, nanoalloys, core-shell 